\title{
USING A SIMULATION MODEL TO COMPARE METHODS OF TREE- RING DETRENDING AND TO INVESTIGATE THE DETECTABILITY OF LOW-FREQUENCY SIGNALS
}

\author{
ANDREW G. BUNN, ${ }^{1, *}$ TIMOTHY J. SHARAC, and LISA J. GRAUMLICH \\ The Big Sky Institute \\ 106 AJM Johnson Hall \\ Montana State University \\ Bozeman, MT USA
}

\begin{abstract}
We use a simulation model to generate tree-ring like data with systematic growth forcings and subject it to two methods of standardization: Regional Curve Standardization (RCS) and Negative Exponential Curve Standardization (NECS). The coherency between very low frequency forcings (hundreds of years) and the chronologies was higher when RCS was used to detrend the component series. There was no difference between standardization methods at decadal or annual time scales. We found that the detectability of systematic forcings was heavily dependent on amplitude and wavelength of the input signal as well as the number of trees simulated. These results imply that for very long tree-ring chronologies where the analyst is interested in low-frequency variability, RCS is a better method for detrending series if the requirements for that method can be met. However, in the majority of situations NECS is an acceptable detrending method. Most critically, we found that multi-centennial signals can be recovered using both methods.
\end{abstract}

Keywords: Dendrochronology, dendroclimatology, Regional Curve Standardization, simulation modeling, low-frequency variation, detrending.

\section{INTRODUCTION}

Despite the importance of tree-ring chronologies as annually resolved quantitative paleoclimate proxies, consensus does not exist among dendrochronologists regarding the best methods for transforming many individual increment cores into mean-value chronologies of the sort used in countless studies. The method by which tree-ring series are processed has a tremendous impact on the mean value chronology of growth (Cook and Kairiukstis 1990). An area of active research in the global change community is identifying the scales and amplitudes of low-frequency variations in temperature in which tree-ring data play a pivotal role (Jones et al. 1998). This is despite claims that tree-ring records cannot provide accurate data on systematic trends in the environment lasting mul-

\footnotetext{
${ }^{1}$ Woods Hole Research Center, P.O. Box 296, Woods Hole, MA 02543-0296.

*Corresponding Author: abunn@whrc.org
}

tiple centuries (Broecker 2001). Were one method of tree-ring processing able to preserve true exogenous low-frequency forcings, say as the result from climate change, then it would logically become the preferred method for working with longlived climate sensitive trees.

A recent report by Esper, Cook, and Schweingruber (hereafter referred to as ECS) documents a new record of extratropical Northern Hemisphere temperature variability with substantially higher multi-centennial variability than previously shown (Esper et al. 2002). The tree-ring based reconstruction of has been compared to the widely cited multi-proxy Northern Hemisphere temperature reconstruction by Mann, Bradley, and Hughes and hereafter referred to as MBH (Mann et al. 1998, 1999). The ECS record shows greater low-frequency variability than the MBH record resulting in discrepancies regarding the magnitude of warming seen about 1000 A.D. during the so-called "Medieval 
Warm Period" (Lamb 1965; Hughes and Diaz 1994).

The ECS reconstruction partially attributes its low-frequency variation to the method used to standardize the raw ring-width series. The detrending of tree-ring series, i.e. the removal of the natural decrease in ring-widths as the tree ages, is an essential part of dendrochronology and is required in virtually all dendroclimatic studies (Fritts 2001). Traditionally if a series shows a non-linear juvenile growth curve, it is removed via negative exponential curve standardization (NECS) whereby a negative exponential curve is fit to each series and the departures from that fit are retained, in some manner or another (Fritts 2001; and see Cook and Peters 1997 for more information). The ECS reconstruction revives and greatly enhances an old detrending technique now called Regional Curve Standardization (RCS) that aligns all the samples to be included in the chronology by cambial age and developing the detrending curve by averaging their values (Esper et al. 2002). Then departures from that fit are taken from the regional curve for each series (see Methods).

There is circularity in asserting which detrending method better reproduces a low-frequency climate signal given that sample series are being used to infer the absolute true signal. Given that dilemma, we developed a simple statistical model of tree growth with known systematic and random noise, and simulated "climate regimes" with known statistical properties. Because the systematic signals are removed from any proximate climate forcing we prefer to call them "growth regimes." We used a simulation framework to build thousands of chronologies under different climatic forcings and two different detrending methods. Our objectives were to compare the efficacy of NECS and RCS in retaining the input signals and to explore what combination of growth forcings lead to the detectability of multi-centennial signals in the simulated trees.

\section{METHODS}

We developed a simulation model to test detrending efficacy and explore issues surrounding low-frequency variability based on known growth parameters for foxtail pine (Pinus balfouriana Grev. et Balf.). Foxtail pine is a high elevation, temperature-sensitive species from the Sierra Nevada, USA that has been critical in many paleoclimatic studies and has shown substantial low-frequency variation (Graumlich 1991; Graumlich 1993; Scuderi 1993; Lloyd and Graumlich 1997). All growth and chronology parameters for the model were built using existing foxtail pine cores from Graumlich's unpublished data.

A statistical simulation model (RingSim) was developed in the $\mathrm{R}$ statistical programming language and environment (Ihaka and Gentleman 1996). All analysis was done in $\mathrm{R}$ using the core functions plus the Rwave contributed package (Carmona et al. 1998). We generated tree-ring like series, with specified features of tree growth, added systematic climatic effects to them, then detrended the series by RCS and NECS. We iterated these steps with random selections of parameters and computed bootstrapped confidence intervals of statistical comparisons of the detrending methods. These steps are described in detail below and a sample run is shown in Figures 1-3.

\section{Generating the Samples}

For each model run, trees were grown in an empty chronology space spanning 2000 years. The ages of the trees ranged from 300 to 1,700 years with a mean age of 550 years and a standard deviation of 250 years fit from a normal distribution. Constraining the tree ages in this manner fits with existing datasets and produces a positively skewed age class distribution. Given that long tree-ring chronologies are composed of living and dead trees, one third of the trees floated in the chronology space similar to incorporation of snags and subfossil wood via cross-dating. The dead trees were fit into the chronology space randomly while the live trees died in the 2,000th year and were germinated (plus years to breast height) in the year that corresponded to 2,000 minus their age. Therefore, a 350 year-old live tree lived in the chronology space from 1650 to 2000 whereas a 350 yearold dead tree could float in the chronology space anywhere that its age permitted (e.g. from 200 to 


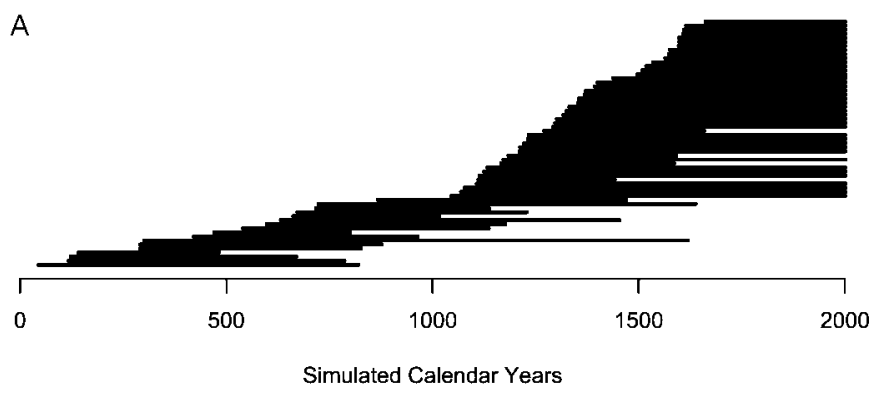

B

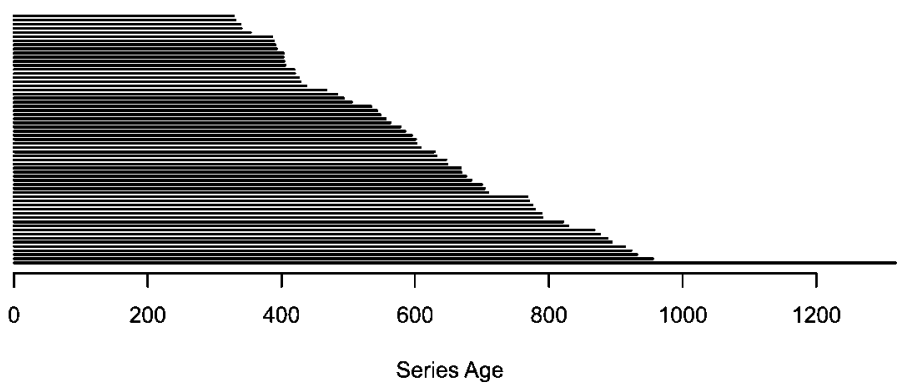

C

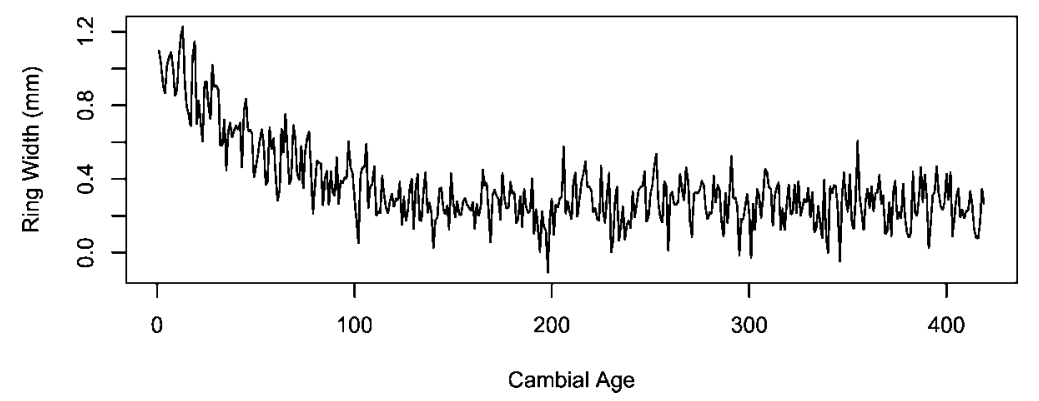

Figure 1. A single run from RingSim has been extracted at random. This model run contains 61 series. It has a multi-centennial period of 229 years, a decadal period of 27 years, and an annual period of 6 years. The amplitudes of the signals are 1.336 , 1.027, and 1.911 respectively. The location of each of the 61 series in the simulated space are shown (A) and reordered in an age class plot (B). One of the series has been plotted at random in the bottom panel (C).

550). The age class structure and floating series plots from a single iteration are detailed in Figure 1.

Parameters for a juvenile growth curve for the simulated series were generated from 53 foxtail pine cores where the pith was clearly visible using cores from Graumlich's lab. The growth curve was then estimated using a negative exponential growth equation $R W_{t}=A e^{-B t}+K$ where $\mathrm{RW}_{t}$ is 
Table 1. Variables used in the 10,000 random model runs. Random numbers for each run were drawn from a random uniform distribution.

\begin{tabular}{llc}
\hline \multicolumn{1}{c}{ Variable } & Abbreviation & Range \\
\hline Initial Ring-Width (mm) & $\mathrm{A}$ & 0.4 to 1 \\
Decay Coefficient & negB & -0.041 to -0.017 \\
Mean Growth at Asymptote & $\mathrm{K}$ & 0.1 to 0.9 \\
Number of Trees & nTrees & 15 to 99 \\
Multi-centennial Period & Period.MC & 200 to 1,000 \\
Decadal Period & Period.Dec & 10 to 60 \\
Annual Period & Period.An & 2 to 10 \\
Multi-centennial Amplitude & Amp.MC & 1 to 1.333 \\
Decadal Amplitude & Amp.Dec & 1 to 1.333 \\
Annual Amplitude & Amp.An & 1 to 1.333 \\
\hline
\end{tabular}

the expected growth at time $t, A$ is the initial year, $-B$ is the slope of the decrease in growth of the ring-widths, $t$ is time in years, and $K$ is the sequential growth per year after the initial growth spurt. Parameters $A,-B$ and $K$ were drawn from a random uniform distribution following the range observed from the cores. Once the growth curve had been created, a simulation from an ARMA model with standard deviations ranging from 0.025 to $0.075 \mathrm{~mm}$ was used to create individual variation in growth with a lag of one year such as those seen from internal gap dynamics [AR1 = $0.2]$. The curve and the temporally autocorrelated noise represent the base growth pattern for each individual series. A single series chosen at random from a random model run is shown in Figure 1C. For each model run the number of trees generated ranged uniformly from 15 to 99 . This ensured that we had many model runs with a low number of trees in the chronology population and many runs with a high number of trees.

\section{Adding the Effects of Climate and Weather}

Two perturbations were created in the chronology space that were added to each individual series. The first was random noise common to all trees-not unlike the effects of interannual weather. Because trees experience weather events slightly differently, a small random scalar was added to the "weather noise" when it was applied to each series. This feature was also an AR1 process at 0.2 and simulated as above to mimic climatic influ- ences, such as temperature variability, which show interannual persistence.

The major feature generated in the chronology space corresponds to long-term climate. Because our objectives are to probe the nature of low frequency signals, the climate regimes generated all contained quasi-oscillatory signals with greater than 200 year periods. Because of the possible interaction of multiple scales of climate forcing we added two more types of signal at the lower orders of magnitude: years and decades. The multi-centennial forcing ranged from 200-1,000, the multidecadal forcing ranged from 20-60 years, and the annual forcing ranged from 2-10 years (Table 1). Magnitudes for observed and modeled forcings were much scarcer in the literature (particularly for the lower-frequency forcings) and we set their sum influence on the series to range uniformly from zero to two (removing to doubling the growth for year $t$ ). That is, each forcing was a scalar that ranged from a minimum of 0.66 to a maximum of 1.33 so that the sum forcing of all the forcings could double or negate the growth for any year $t$ if the waves were all at the extent of the range and in phase.

The growth forcing was generated using an inverse Fourier transform. For each run, a Fourier space was generated for 2,000 years (the maximum length of the chronologies). Three spaces in the Fourier space corresponding to the frequencies drawn for the model run were filled with random normal numbers with a mean of zero and a standard deviation equal to the amplitudes drawn for 

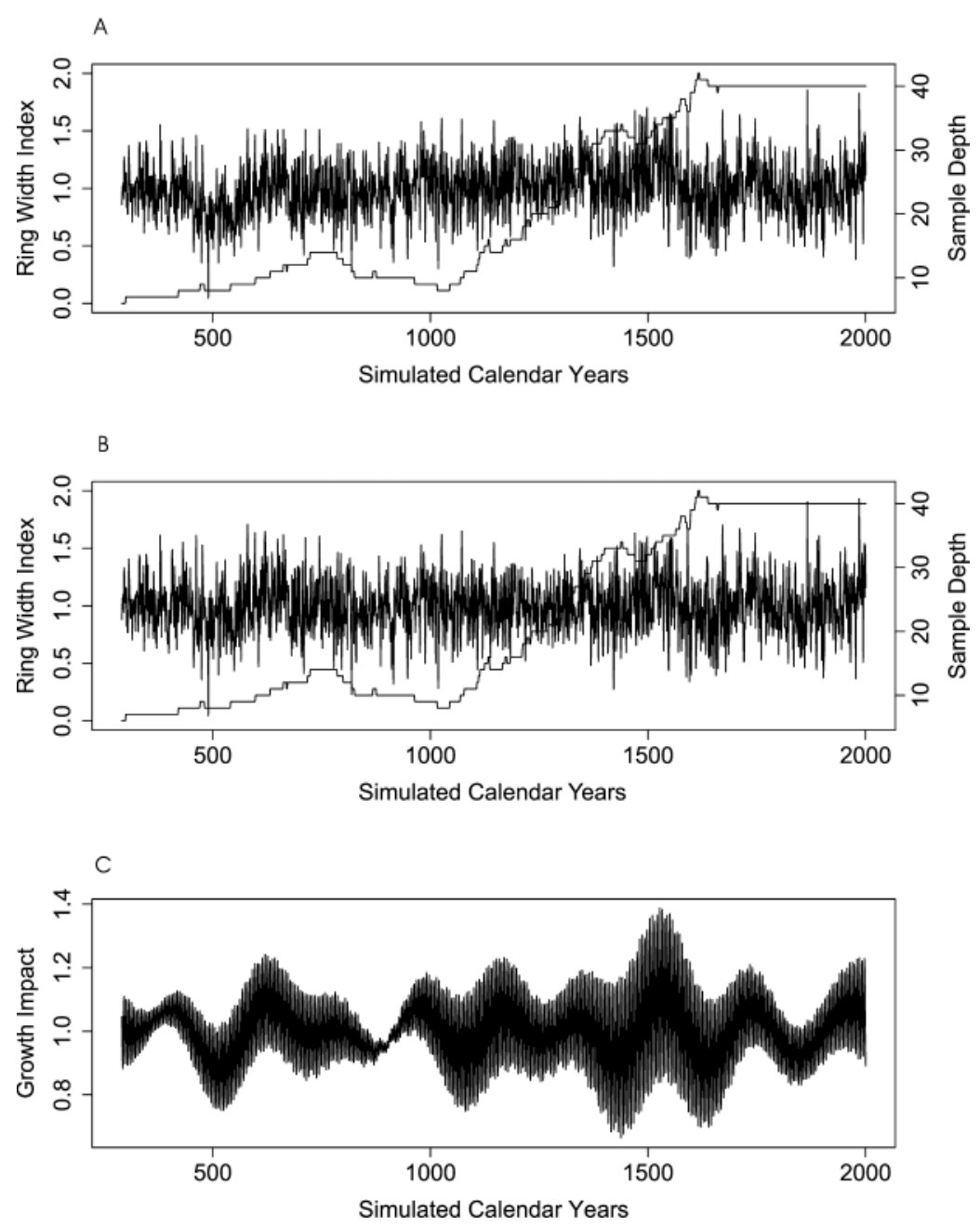

Figure 2. The RCS (A) and NECS (B) chronologies are shown along with the growth regime (C) from the run depicted in Figure 1. The sample depth is plotted alongside the chronologies (A \& B).

the corresponding frequency. Finally, this complex vector was transformed out of Fourier space. The result is an oscillating wave with known periods and amplitudes (see Figure 2C). Each series was multiplied by this wave and therefore incorporated this systematic noise.

\section{Detrending and Chronology Construction}

ECS built two chronologies with RCS-one with the series demonstrating non-linear trends and one with series demonstrating linear trends and observed only slight differences between them. Nonlinear series are the biological expectation and more challenging to detrend than linear series. Therefore, our simulated data are exclusively nonlinear (Table 1) so as to provide a more conservative test of the detrending methods. We followed the methods of Esper et al. (2003) in developing the regional curve. The regional curve was calculated by aligning each series by cambial age and taking the bi-weight robust mean for a given age (Cook 1985). A cubic spline was fit to the mean values with a $50 \%$ frequency cut-off width equal to $10 \%$ of the length (Cook and Peters 1981; and see Cook and Kairiukstis 1990 for more information on smoothing splines). A ring-width index (RWI) measured departures from the regional 
curve and was calculated as ratios (Fritts 2001). We also explored the use of residuals and residuals with a power transformation (Cook and Peters 1997). As noted by Esper et al. (2003) neither residuals nor ratios can be universally recommended for millennial-length chronologies. We found that the results were often, but not always, similar with all three methods and plan to systematically explore the differences in future work. A chronology of all the series was built by taking the average for each year. The minimum number of series typically used in chronologies varies depends on the strength of the expressed climate signal across cores, their fractional variance terms, and the desired accuracy level (Wigley et al. 1984). Based on previous experience with the sub sample signal strength and expressed population signal of foxtail pine (e.g. Graumlich 1991, 1993) we arbitrarily truncated our chronologies to a sample depth of five series to be consistent with the biology of the species we are simulating.

To perform the NECS we used non-linear regression to fit a negative exponential curve to each series. The model was used to predict a growth curve for each series and each series was detrended using the ratio method as above. A chronology was also built as above (e.g. Figure 2A and B). A continuous wavelet transform for all three time series is shown in Figure 3 and shows that both methods faithfully capture most of the true power spectrum for this example (see Torrence and Compo 1998 for a review on wavelet analysis).

The additional power generated by the AR functions can be seen in the wavelet spectra in the highest frequencies. The comparison provided in Figure 3 is also useful for seeing the abundance and diversity of structures that exist in the simulated tree-ring chronologies that are common to both detrending techniques and do not exist in the growth forcing.

Figure 3. Continuous wavelet power spectra density estimates for the RCS (A) and NECS (B) chronologies and the growth regime (C) are shown. The gray-scaled contour intervals show $0-25 \%, 25-50 \%, 50-95 \%, 75-95 \%$, and $95-100 \%$ of the wavelet power. There are few differences between the chronologies and their coherence with the growth regime.

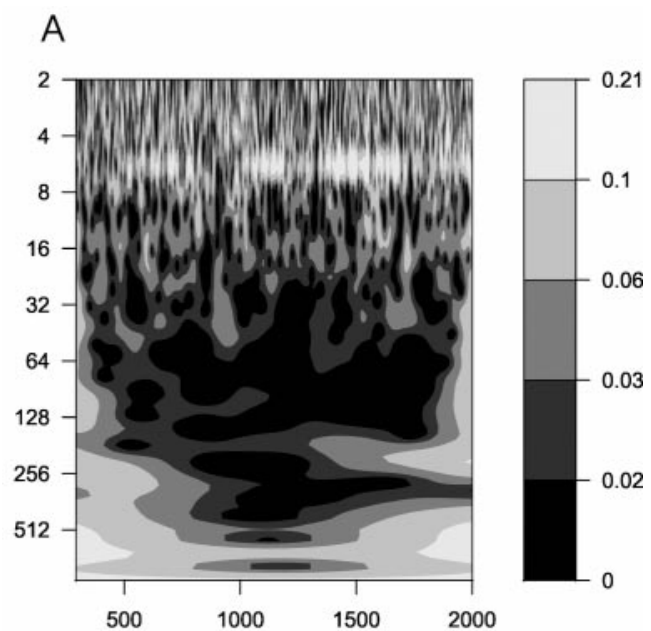

B
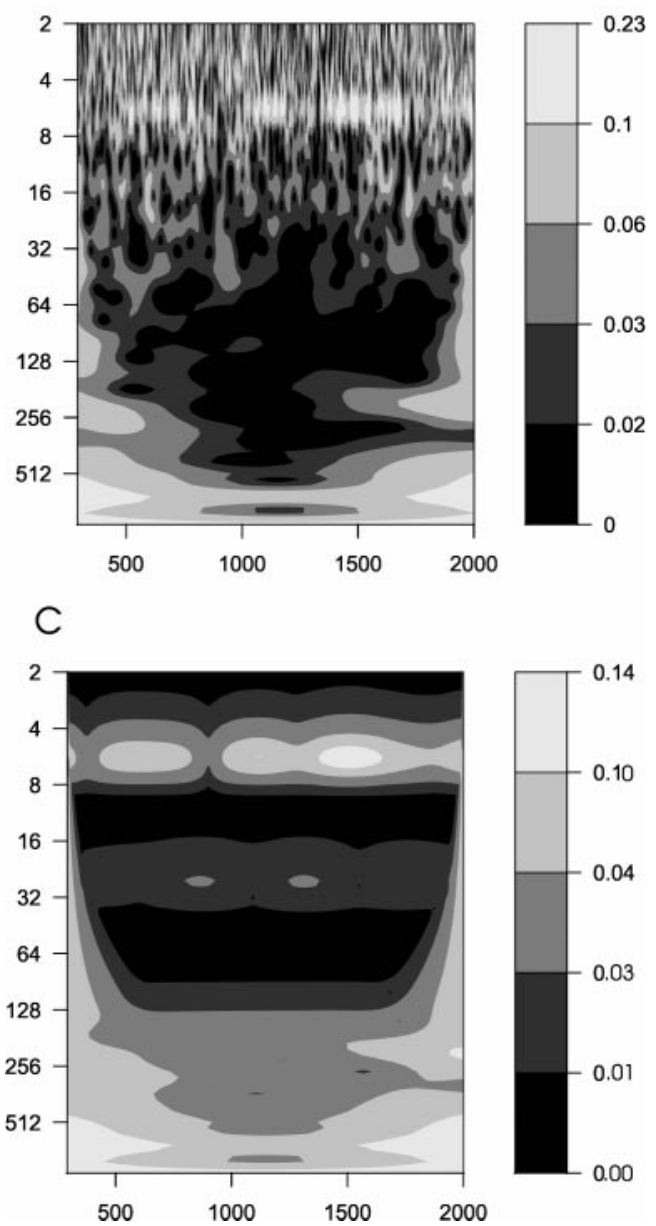
Table 2. Example output for two of 10,000 model runs. The number of trees simulated is followed by the inputs for the growth forcings. The squared coherency between each standardized chronology and the climate disturbance is shown for each time scale examined. $\mathrm{nTrees}=$ Number of Trees; $\mathrm{MC}=$ Multi-centennial; Dec $=$ Decadal; An $=$ Annual.

\begin{tabular}{|c|c|c|c|c|c|c|c|c|c|c|c|c|c|}
\hline \multirow[b]{2}{*}{ ID } & \multirow[b]{2}{*}{ nTrees } & \multicolumn{3}{|c|}{ Periods } & \multicolumn{3}{|c|}{ Amplitudes } & \multicolumn{3}{|c|}{ Coherency for RCS } & \multicolumn{3}{|c|}{ Coherency for NECS } \\
\hline & & $\mathrm{MC}$ & Dec & An & $\mathrm{MC}$ & Dec & An & $\mathrm{MC}$ & Dec & An & $\mathrm{MC}$ & Dec & An \\
\hline 1 & 39 & 836 & 25 & 7 & 1.128 & 1.106 & 1.256 & 0.894 & 0.820 & 0.995 & 0.814 & 0.780 & 0.995 \\
\hline 2 & 56 & 536 & 28 & 3 & 1.183 & 1.061 & 1.139 & 0.982 & 0.643 & 0.992 & 0.949 & 0.693 & 0.990 \\
\hline
\end{tabular}

Assessing Standardization Efficacy with Model Runs

With a chronology built using RCS and NECS the model is then able to compare each chronology to the systematic growth input. The RCS chronology, the NECS chronology and the systematic growth inputs were combined into a multivariate time series and run through a spectral analysis (see Brockwell and Davis 1991 and Venables and Ripley 2002 for good reviews of spectral analysis). Standardization efficacy was assessed using the squared coherency of the time series. Coherency analysis evaluates the correlation of multiple time series at all frequencies and is reported in terms of magnitude-squared coherence $[0,1]$. We judged how well the chronologies captured the growth signal by looking at the squared coherency of between the input growth signal and the two types of chronology at the exact frequencies used to generate the growth signal.

We ran the model 10,000 times. At each iteration, there were randomly assigned values for growth-regime periods, growth-regime amplitudes, biological growth factors, number of trees per chronology, and random noise as described above. The number of trees used in the simulation, the growth-regime periods and amplitudes, and the coherencies between the growth-regime forcings and each type of chronology for the three time scales examined were saved. Table 2 contains an example of two iterations of model output. Because the coherency output from the model is not normally distributed, we used a non-parametric bootstrap test to compare the difference in the means of the coherencies for RCS minus NECS (i.e. $\left(\sum_{i=i}^{n}\right.$ Coh$\left.R C S_{i} / n\right)-\left(\sum_{i=i}^{n}\right.$ CohNECS $\left._{i} / n\right)$ where CohRCS is the squared coherency for the RCS chronology, CohNECS is the squared coherency for the RCS chronology at run $i$, and $n$ is the 10,000 model runs-see Davison and Hinkley (1997) and Venables and Ripley (2002) for more information.

\section{Results}

The RCS method shows significantly greater ability to retain low frequency information as compared to the NECS method (Table 3). The difference in the means of the multi-centennial coherencies for RCS and NECS is 0.048. Studentized 95\% confidence intervals from 1,000 bootstrap replicates with 100 internal bootstrap replications are 0.042 and 0.054 . Because the $95 \%$ CIs do not span zero, the null hypothesis that there is no difference between the means is rejected and therefore the difference between RCS and NECS in retaining low-frequency signals is statistically sig-

Table 3. The mean coherency between the standardization methods (RCS and NECS) and the systematic noise are shown for each time scale. The difference in the means values of RCS and NECS are significant from zero under a studentized bootstrapped resampling test $(\mathrm{R}=1,000, \mathrm{M}=100)$ for the multi-centennial periods only.

\begin{tabular}{lcccr}
\hline Temporal Scale & RCS Coherency & NECS Coherency & $\begin{array}{c}\text { Mean(RCS) }- \\
\text { Mean(NECS) }\end{array}$ & \multicolumn{1}{c}{$95 \%$ CI } \\
\hline Multi-centennial & 0.848 & 0.800 & 0.048 & $0.042-0.054$ \\
Decadal & 0.895 & 0.902 & -0.007 & $-0.013-0.003$ \\
Annual & 0.908 & 0.914 & -0.006 & $-0.011-0.002$ \\
\hline
\end{tabular}



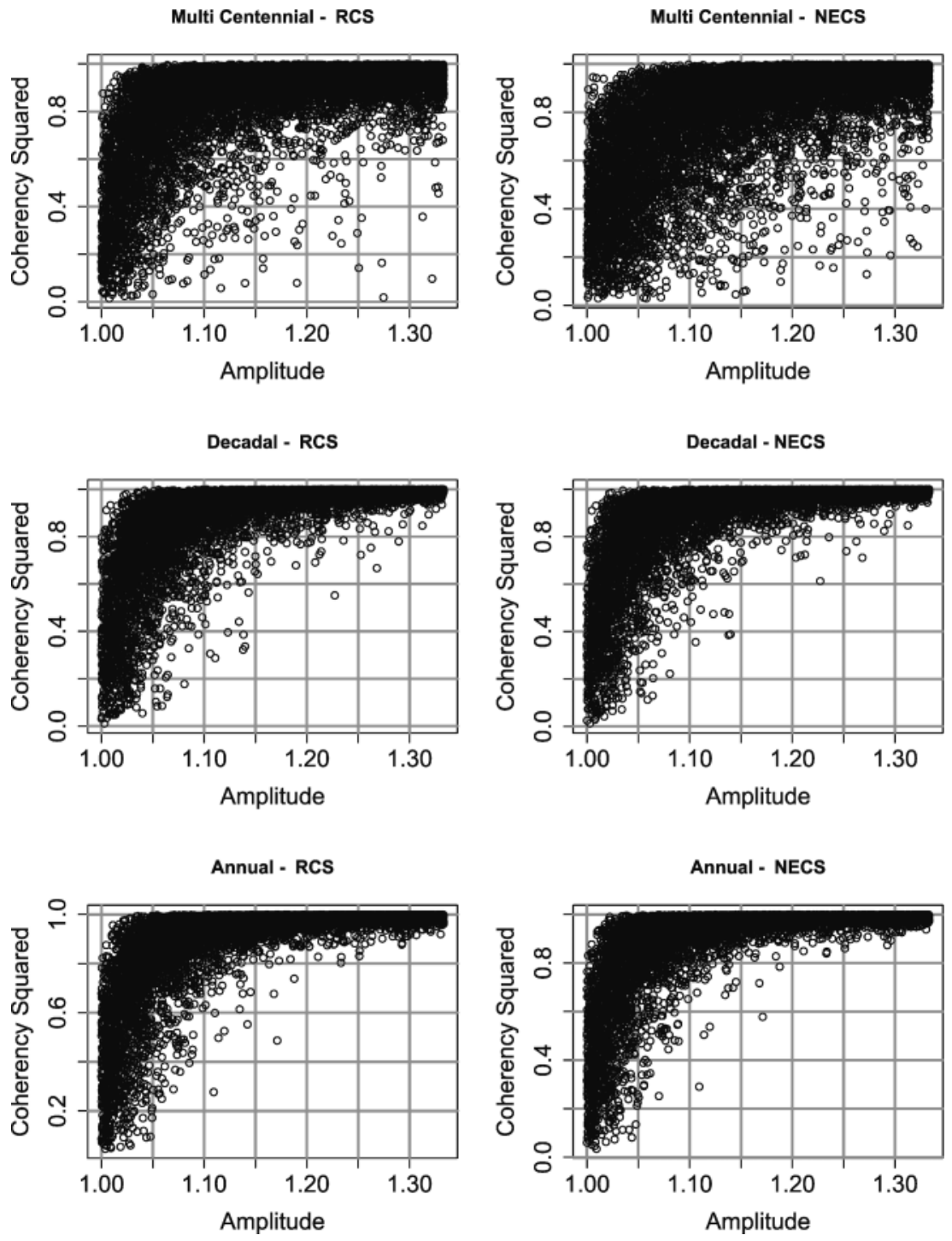

Figure 4. Coherency squared between the growth regime and chronology is shown as a function of signal amplitude for RCS and NECS chronologies (columns) for the three time scales examined (rows). The only noticeable difference between the methods is at the multi-centennial time scale (compare to Table 3).

nificant. The differences in the means between the RCS method and the NECS method in retaining higher frequency signals corresponding to decadal and annual periodicities are not significantly greater than zero (Table 3).

The ability to detect systematic signals in the chronologies is easily seen as a function of the input signal's amplitude. Signals less than 1.06 are difficult to detect, regardless of period (Figure 4).
Furthermore, at amplitudes greater than 1.13 the RCS chronologies were more coherent with the input than the NECS chronologies at the multicentennial time scale (Figure 4, top). When decomposed by period and amplitude of the input signal, RCS begins to outperform NECS at periods greater than 450 years (Figures $5 v s .6$ ). The differences between RCS and NECS are not detectable at decadal or annual time scales. 


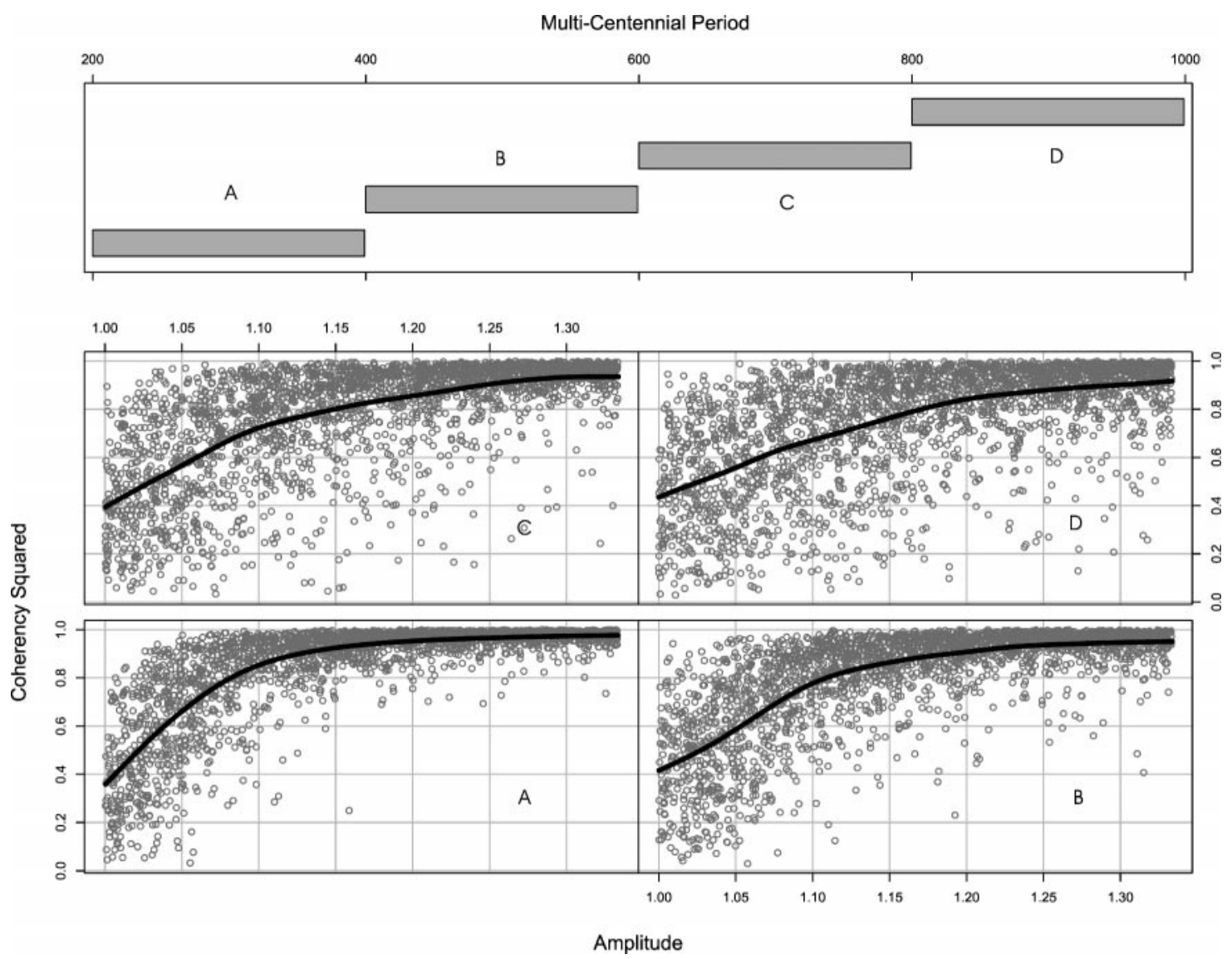

Figure 5. A conditioning plot shows the coherency squared between the growth regime and the RCS chronology as a function of signal amplitude conditioned by the multi-centennial period. The bottom left panel (A) subsets model runs with periods between 200 and 400 years while the top right panel (D) subsets model runs with periods from 800 to 1,000 years. There is minimal loss of coherency at longer wavelengths. The black lines are stiff cubic splines to highlight trends in the data.

The RCS method is sensitive to the number of series used to generate the regional curve. If the model runs containing 15 trees are subset (115 of the 10,000 models runs), then the mean coherency between the input signals and the RCS chronology for the multi-centennial time scales is 0.76 . The mean coherency between the input signals and the NECS chronology for the multi-centennial time scales is 0.79 for the same data. This is contrary to the entire dataset where RCS coherency is greater than NCS at multi-centennial time scales. However, the difference in the means of the squared coherencies (RCS minus NECS) for the subset containing 15 trees is not significantly different from zero under the same bootstrap procedure de- scribed above. This fails to reject the null hypothesis that there is no difference between the means of the squared coherency for RCS and NECS for model runs containing 15 trees. At the other end of the spectrum the general relationship holds. If the model runs containing 99 trees (117 of the 10,000 models runs) are subset, then the mean coherency between the input signals and the RCS chronology for the multi-centennial time scales is 0.90 . The mean coherency between the input signals and the NECS chronology for the multi-centennial time scales is 0.81 for the same data. The relationship between the number of trees in the chronology, amplitude, and multi-centennial period for the RCS method is illustrated in Figure 7. 


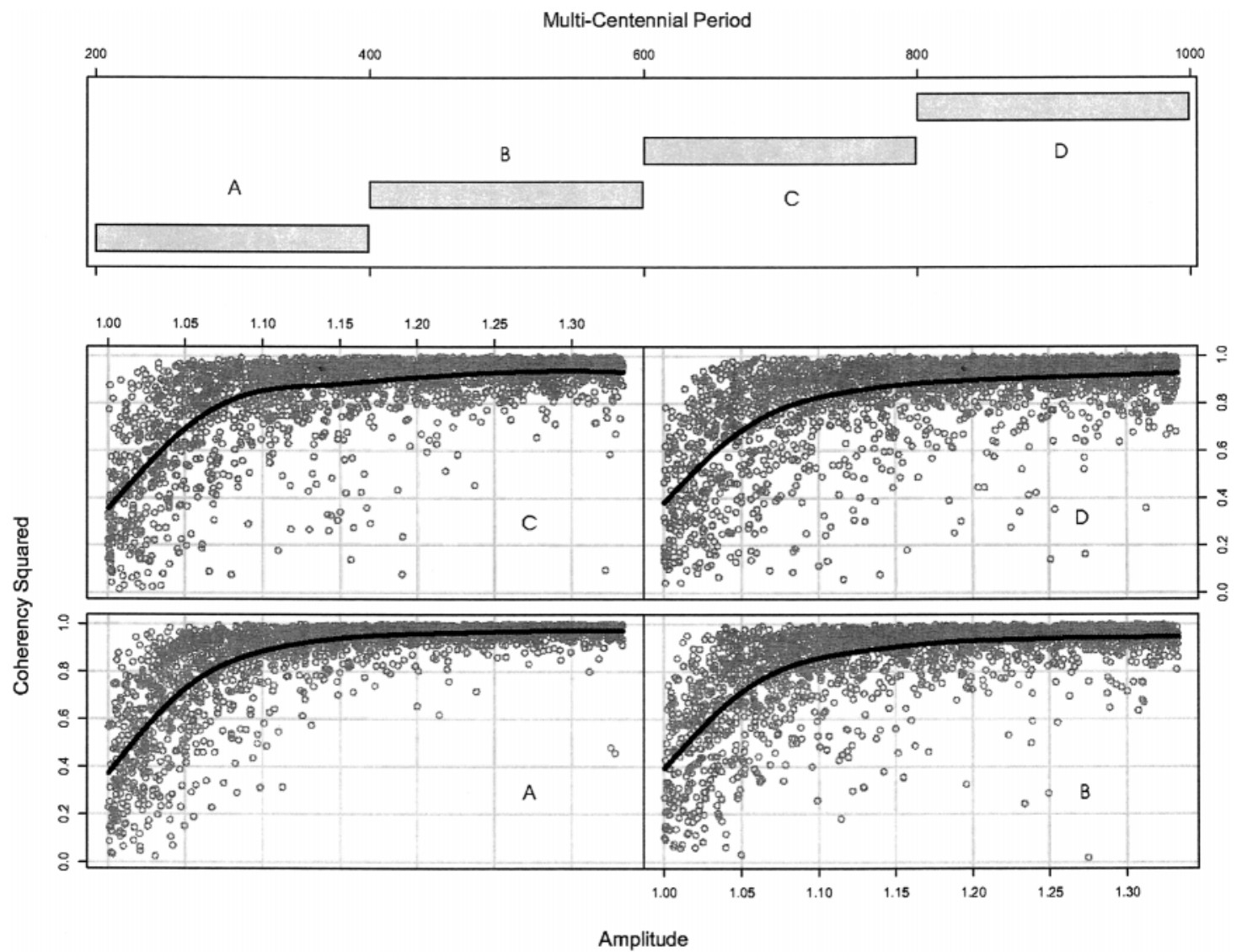

Figure 6. A conditioning plot shows the coherency squared between the growth regime and the NECS chronology as a function of signal amplitude conditioned by the multi-centennial period. As above, the bottom left panel (A) subsets model runs with periods between 200 and 400 years while the top right panel (D) subsets model runs with periods from 800 to 1,000 years. Unlike the RCS chronologies, the NECS shows substantial degradation of coherency at the lowest frequencies even at the strongest amplitudes.

\section{DISCUSSION}

Regional curve standardization outperforms negative exponential decay standardization for maintaining low-frequency variability in our simulated tree-ring chronologies but does not preserve decadal or annual periods with greater efficacy. This supports previous claims regarding the powers of RCS in maintaining low-frequency variability (Briffa et al. 1992; Briffa et al. 1996; Esper et al. 2002, 2003). This also contributes a methodological basis to the body of literature that refutes the idea that tree-ring chronologies cannot preserve low-frequency signals (e.g. Esper et al. 2002). Despite these results we concur with Briffa et al. (1996) who state, "The RCS approach is far from being a general panacea for the loss of longtimescale information in dendroclimatology," and discuss the results and caveats in our model.

There are enormous advantages to using a simulation model to explore questions of standardization and low-frequency signals. All the advantages correspond to the natural variation in the parameters examined: tree-ring series show variation in juvenile growth curves; tree-ring chronologies have variation in sample depth; climate periods vary at multiple scales. Even for a model as simple as this one the parameter-space for these questions is enormous (Table 1). The simulation framework allows us to explore the response variable, coherency of a chronology to a known growth modifier, 


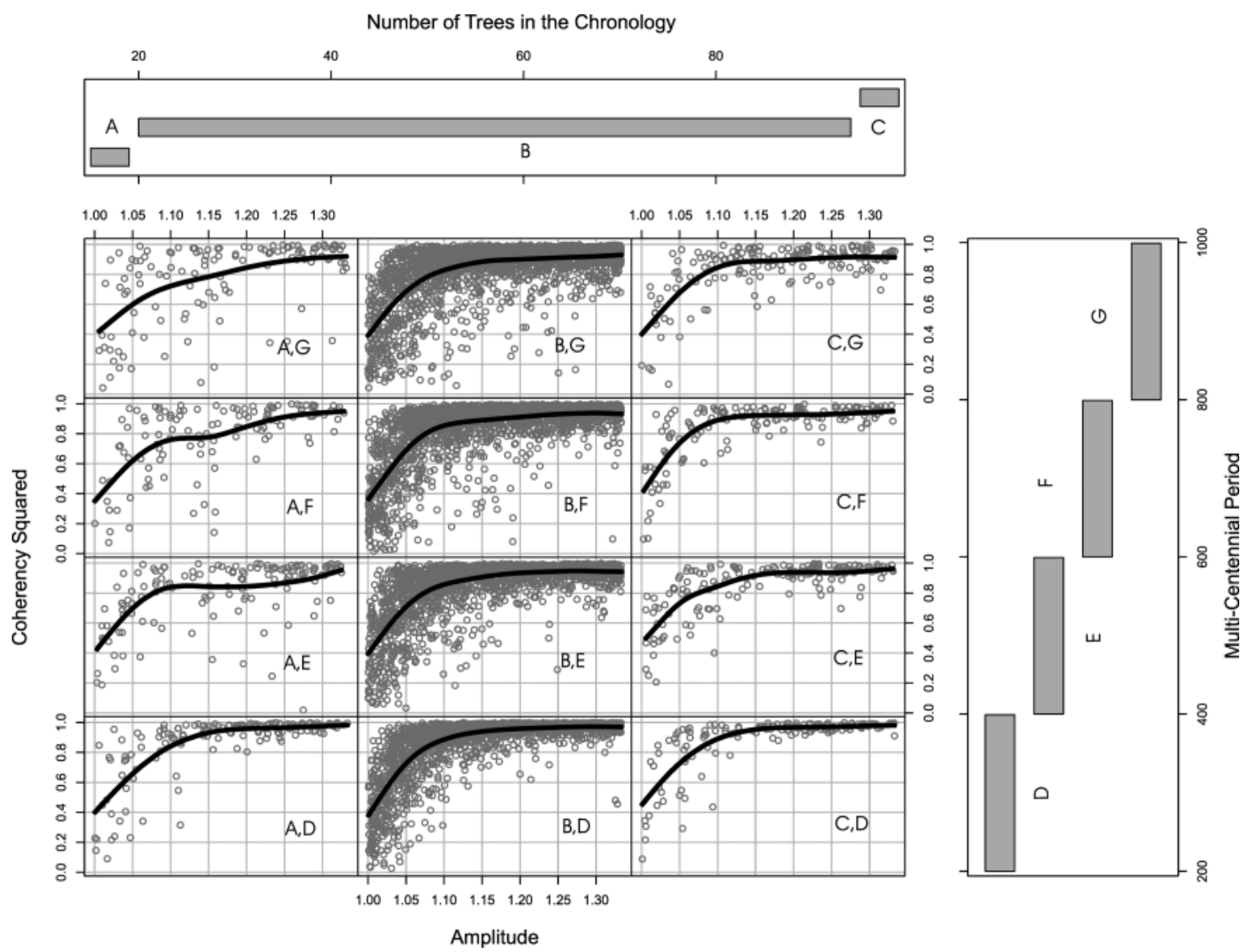

Figure 7. A double conditioning plot shows the coherency squared between the growth regime and the RCS chronology as a function of signal amplitude conditioned by the multi-centennial period (rows) and the number of trees used in the chronology (columns). For instance, the top left panel (A, G) subsets model runs with 15 to 20 trees and periods from 800 to 1,000 years. The bottom right panel (C, D) subsets model runs with 95 to 99 trees and periods from 200 to 400 years. This shows the effect of the number of trees on the detectability of multi-centennial periods where the longest periods are easiest to detect with the highest number of trees. However, at high enough amplitudes signals are generally detectable regardless of period or the number of trees. The NECS method is not shown because the difference between the numbers of trees was not as striking.

with well-enumerated predictors (e.g. the number of trees simulated). The advantage of establishing this model with minimal assumptions (e.g. using uniform distributions for selecting parameters) will allow us to perform uncertainty and sensitivity analysis in future work.

Our tree growth simulation model is statistical, meaning that model parameters for growth and forcings are not directly derived from the proximate causes of those variables. Biologically, the width of the initial ring of a foxtail pine core is a function of air and soil temperatures and soil water content for periods of the tree's growing season among other factors. In our model, the width of the initial ring of a series is drawn from a random distribution of observed initial widths. Similarly, the periods of growth-regime variability are drawn from observed periodicities and removed from first-order calculations of sea surface temperatures, orbital parameters, etc. These gross generalizations allow us to explore the relationship between signal duration and strength from theory without becoming mired in the contentious and highly technical aspects of climate forcings and climate-growth relationships over time.

In the model, trees are grown following a negative exponential curve that varies with parameters observed in cores from foxtail pines. Tree growth 
is idealized using a negative exponential curve with three parameters: the initial width of the first ring, the mean growth after the juvenile period and a coefficient that determines the rate of decay from the initial width to the mean adult width. To avoid circularity when the series were detrended, these parameters were estimated without the aid of a mathematical model. However, some circularity was introduced when the series were generated in the model which necessitated using a negative exponential function. In view of the fact that NECS has an advantage (the growth curves were generated with a negative exponential) it is significant that the RCS detrending method was better at extracting the low-frequency signal.

The RCS method is an appealing theoretical framework for removing low-frequency signals from tree-ring series that are much shorter than the periods being sought. The reasons for this have been well-covered elsewhere (Briffa et al. 1996; Esper et al. 2003) and will not be discussed here beyond pointing out that negative exponential curves are extremely sensitive to the first decades of growth. For instance, if a series originates during a period of high growth (e.g. favorable climate) the curve fit to that via NECS will dampen that signal by misfitting the initial growth parameter and decay coefficient. The RCS method guards against this if the series are distributed across a variety of growth periods (i.e. some series originate in periods of favorable growth and some periods initiate in periods of restricted growth).

Having series distributed across a range of growth conditions is necessary but not sufficient in developing an accurate regional curve. Our model results also indicate that RCS is sensitive to the number of series used to develop the regional curve. The general relationship for all model runs is for RCS to be more coherent with low-frequency signals (Table 3). That relationship does not hold when the number of trees in the chronologies ranges equals 15 . In those cases, NECS and RCS are statistically equivalent. Although our results indicate that there is no statistical difference between RCS and NECS for a chronology with 15 trees, we are reluctant to give a recommendation on the number of series needed for an accurate regional curve given the tendency of dendrochro- nologists to use multiple series from a single tree and the difficulty of aligning real tree-ring series by cambial age. Constructing a regional curve requires the analyst to know the correct cambial age of the series. In a simulation model, this is known. In ancient tree-ring series and sub-fossil wood samples, the pith is often missing caused by rot, requiring the application of pith offsetting to estimate cambial age. Although Esper et al. (2003) expend considerable energy exploring regional curves where the pith of some series was estimated and report good results, caution is still warranted. The construction of a good tree-ring chronology requires the analyst to make judgments about which cores (or portions of a core) to include. This is especially true with very long, temperature-sensitive chronologies. We do not want a researcher to work towards a threshold of samples that are indicated by this model but instead urge the careful consideration of each sample and the exploration of both detrending methods.

RCS shows improvement over NECS at multicentennial scales only (Table 3, Figure 4). At the multi-centennial scale, RCS shows the greatest improvement at the longest wavelengths (Figures 5 and 6). These wavelengths are thought to be expressed in temperature variability (e.g. Jones et al. 1998; Mann and Jones 2003) indicating that when low-frequency temperature variability is being investigated, and when the data support the construction of an accurate regional curve, RCS is to be preferred over NECS. The analyst should also consider using multiple methods of detrending. For instance in real tree-ring series, high-frequency signals tend to be local compared to multi-decadal or centennial signals (Jones et al. 1998). In those cases, a NECS chronology could be used to display and interpret high-frequency features in a chronology while RCS could be used to emphasize low-frequency features. As is common in science, one method will rarely accomplish all the research goals.

The retrieval of an input signal was highly dependent on its amplitude. Signals were difficult when the scalar applied was less than 1.06. This occurs when the signal falls below the background noise simulated in the chronology. In a simulated chronology the amount of noise from day-to-day 
weather and stand dynamics is known. In real treering data the level of background noise (red or white) is troublesome to remove without affecting the true signals being sought. Dendroclimatic research therefore always seeks systems where the interference of non-climatic signals can be minimized (e.g. latitudinal and alpine treeline). A significant challenge to the global change community is to quantify unbiased estimates of the amplitude of the climate signal to tree growth (transfer function) as well as the amount of background noise inherited by each series. If that becomes possible, estimates about the relative detectability of lowfrequency climate signals can be drastically improved.

\section{CONCLUSIONS}

Of the more than 2,400 entities in the International Tree-Ring Database, roughly 5\% are greater than 800 years in length. Only a fraction of these chronologies are of interest to researchers extracting low-frequency variation related to climate. According to our model results, low-frequency variability, of the sort seen in the ECS reconstruction of extra-tropical tree-rings, is better preserved using RCS if an accurate regional curve can be constructed. This in no way diminishes the multiproxy $\mathrm{MBH}$ reconstruction but rather points to the need to further understand the spatial scale of the expression of low-frequency variation. Most importantly it shows that low frequency variation can be preserved in tree chronologies. Given the paucity of very long tree-ring chronologies, proper processing techniques are critical and the RCS method shows tremendous promise.

\section{ACKNOWLEDGMENTS}

The Canon National Parks Science Scholars Program supports Bunn's research along with the Sierra Nevada Global Change Project under USGS contract CA99WRAG0026 to Graumlich. The Montana State University Undergraduate Scholar's Project generously supported Sharac during this research. Jeremy Littell, Steve Gray, and Greg Pederson all gave helpful comments on this manuscript.

\section{REFERENCES CITED}

Briffa, K. R., P. D. Jones, T. S. Bartholin, D. Eckstein, F. H. Schweingruber, W. Karlen, and P. Zetterburg

1992 Fennoscandian summers from AD 500: Temperature changes on short and long timescales. Climate Dynamics 7:111-119.

Briffa, K. R., P. D. Jones, F. H. Schweingruber, W. Karlén, and S. G. Shiyatov

1996 Tree-ring variables as proxy-climate indicators: Problems with low-frequency signals. In Climatic Variations and Forcing Mechanisms of the Last 2000 Years, edited by P. D. Jones, R. S. Bradley, and J. Jouzel, pp. 9-41. Springer-Verlag.

Brockwell, P. J., and R. A. Davis

1991 Time Series: Theory and Methods. Second edition. Springer Verlag, New York.

Broecker, W. S

2001 Was the medieval warm period global? Science 291: 1497-1499.

Carmona, R., W. L. Hwang, and B. Torresani

1998 Practical Time-Frequency Analysis: Gabor and Wavelet Transforms with an Implementation in $S$. Academic Press, San Diego.

Cook, E. R.

1985 A Time-Series Analysis Approach to Tree-Ring Standardization. Ph.D. dissertation. The University of Arizona, Tucson.

Cook, E. R., and L. A. Kairiukstis

1990 Methods of Dendrochronology. Kluwer Academic Publishers, The Netherlands.

Cook, E. R., and K. Peters

1981 The smoothing spline: a new approach to standardizing forest interior tree-ring width series for dendroclimatic studies. Tree-Ring Bulletin 41:45-53.

Cook, E. R., and K. Peters

1997 Calculating unbiased tree-ring indices for the study of climatic and environmental change. The Holocene 7:361-370

Davison, A. C., and D. V. Hinkley

1997 Bootstrap Methods and Their Application. Cambridge University Press, Cambridge.

Esper, J., E. R. Cook, and F. H. Schweingruber

2002 Low-frequency signals in long tree-ring chronologies for reconstructing past temperature variability. Science 295:2250-2253.

Esper, J., E. R. Cook, F. H. Schweingruber, and K. Peters

2003 Detecting low frequency tree-ring trends by the RCS method. Tree-Ring Research 59:81-98.

Fritts, H. C.

2001 Tree Rings and Climate. Blackburn Press, New Jersey.

Graumlich, L. J.

1991 Subalpine tree growth, climate, and increasing CO2: an assessment of recent growth trends. Ecology 72 : $1-11$. 
Graumlich, L. J.

1993 A 1000-year record of temperature and precipitation in the Sierra Nevada. Quaternary Research 39:249255

Hughes, M. K., and H. F. Diaz

1994 Was there a "Medieval Warm Period" and if so, where and when? Climatic Change 26:109-142.

Ihaka, R., and R. Gentleman

1996 R: a language for data analysis and graphics. Journal of Computational and Graphical Statistics 5:299314.

Jones, P. D., K. R. Briffa, T. P. Barnett, and S. F. B. Tett

1998 High-resolution palaeoclimatic records for the last millenium: Interpretation, integration and comparison with general circulation model control-run temperatures. The Holocene 8:455-471.

Jones, P. D., T. J. Osborn, and K. R. Briffa

1997 Estimating sampling errors in large-scale temperature averages. Journal of Climate 10:2548-2568

Lamb, H. H.

1965 The early medieval warm epoch and its sequel. $P a$ laeogeography, Palaeoclimatology, Palaeoecology 1: 13-37.

Lloyd, A. H., and L. J. Graumlich

1997 Holocene dynamics of treeline forests in the Sierra Nevada. Ecology 78:1199-1210.
Mann, M. E., R. S. Bradley, and M. K. Hughes

1998 Global-scale temperature patterns and climate forcing over the past six centuries. Nature 392:779-787.

Mann, M. E., R. S. Bradley, and M. K. Hughes

1999 Northern hemisphere temperatures during the past millenium: Inferences, uncertainties, and limitations. American Geophysical Union 3:1-8.

Mann, M. E., and P. D. Jones

2003 Global surface temperatures over the past two millennia. Geophysical Research Letters 30:1820-1824.

Scuderi, L. A.

1993 A 2000-year tree ring record of annual temperatures in the Sierra Nevada Mountains. Science 259:14331436.

Torrence, C., and G. P. Compo

1998 A practical guide to wavelet analysis. Bulletin of the American Meteorological Society 79:61-78.

Venables, W. N., and B. D. Ripley

2002 Modern Applied Statistics with S-PLUS. Fourth Edition. Springer, New York.

Wigley, T. M. L., P. D. Jones, and K. R. Briffa

1984 On the average value of correlated time series, with applications in dendroclimatology and hydrometeorology. Journal of Climate and Applied Meteorology 23:201-213.

Received 24 October 2003; accepted 23 July 2004. 